\title{
ADSORÇÃO DOS CORANTES REATIVO PRETO 5 E AZUL DE METILENO POR FIBRAS DE SISAL: APLICAÇÃO DO MODELO HSDM
}

\author{
V. H. VARGAS ${ }^{1}$, R. R. PAVÉGLIO ${ }^{1}$, N.P.G. SALAU ${ }^{1}$, G. L. DOTTO ${ }^{1}$ \\ ${ }^{1}$ Universidade Federal de Santa Maria, Departamento de Engenharia Química \\ E-mail para contato: victoria.hvargas@gmail.com
}

\begin{abstract}
RESUMO - Neste trabalho foi investigado o potencial de adsorção de fibras de sisal na remoção dos corantes azul de metileno (MB) e preto reativo 5 (RB5), em termos da cinética, via aplicação do modelo HSDM. Na cinética de adsorção, para ambos os corantes, o modelo HSDM demonstrou-se apropriado para representar os dados experimentais. Os valores estimados do coeficiente de difusão $(D s)$ foram de $1,6 \times 10^{-11}$ e $5,9 \times 10^{-10} \mathrm{~cm}^{2} \mathrm{~s}^{-1}$ para os corantes preto reativo 5 (RB5) e azul de metileno(MB). As capacidades de adsorção no equilíbrio (qe), para o AM e para o RB5, respectivamente, foram de $87 \mathrm{mg} \mathrm{g}^{-1}$ e $29,8 \mathrm{mg} \mathrm{g}^{-1}$, com percentuais de remoção maiores que $85 \%$. De uma forma geral, observa-se que fibras de sisal são um adsorvente alternativo, podendo ser utilizadas para a remoção de corantes de meios aquosos.
\end{abstract}

\section{INTRODUÇÃO}

Muitos dos problemas de poluição são causados pela produção de resíduos nos processos industriais. Os processos têxteis, em especial, são grandes consumidores de água e de corantes sintéticos, gerando efluentes coloridos de complexa composição, os quais, quando não corretamente tratados, podem causar graves problemas de contaminação ambiental. $\mathrm{O}$ azul de metileno (MB) é um dos corantes mais notórios e amplamente utilizados na indústria, entretanto, segundo Vadivelan e Kumar (2005), o MB possui vários efeitos nocivos sobre a vida humana, podendo causar danos no sistema nervoso e problemas cardíacos. Os corantes reativos, como o reativo preto 5 (RB5), são também muito utilizados nos processos têxteis, devido ao seu simples procedimento de tingimento. Portanto, há a necessidade de tratamento dos efluentes coloridos antes de sua descarga nos ecossistemas. Atualmente, várias técnicas de tratamento vêm sendo estudadas. Os métodos convencionais envolvem oxidação avançada, foto-degradação, tratamento por membranas, tratamento biológico, adsorção, entre outros. Dentre estes, a adsorção é o método que se destaca, devido ao seu baixo custo, simplicidade de aplicação, rapidez e alta eficiência. O carvão ativado é muito utilizado como adsorvente, entretanto, seu alto custo de regeneração faz com que se busque cada vez mais materiais alternativos. Neste contexto, fibras de sisal podem ser utilizadas como adsorvente no tratamento de águas e de efluentes. O presente trabalho teve como objetivo avaliar o potencial de fibras de sisal na adsorção dos corantes Reativo Preto 5 (RB5) e Azul de Metileno (MB), em termos de ensaios cinéticos. As curvas cinéticas foram obtidas considerando um tempo de contato de 0 a 240 minutos, no $\mathrm{pH}$ adequado para cada corante. Os dados resultantes foram ajustados ao modelo HSDM para estimação do coeficiente de difusão $\left(D_{s}\right)$. 


\section{MATERIAIS E MÉTODOS}

\subsection{Preparação e Caracterização das Fibras de Sisal}

As fibras de sisal, Figura 1, foram obtidas em parceria com a Universidade Estadual de Santa Cruz (BA). O material foi moído e peneirado, sendo utilizadas as fibras com tamanho de partícula variando de $325 \mu \mathrm{m}$ a $425 \mu \mathrm{m}$. Utilizou-se a espectroscopia de infravermelho com transformada de Fourier (FT-IR) (Shimadzu, Prestige 21, Japão) para identificar os principais grupos funcionais. As características texturais foram visualizadas por microscopia eletrônica de varredura (MEV) (Jeol, JSM-6610LV, Japão).

Figura 1 - Fibras de Sisal

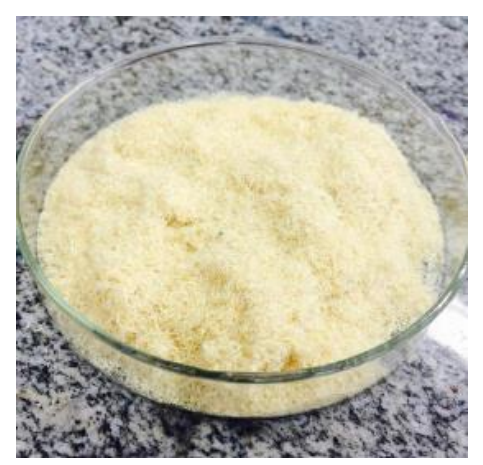

\subsection{Corantes}

Os corantes, Reativo Preto $5\left(991,8 \mathrm{~g} \mathrm{~mol}^{-1}, \lambda_{\max }=597 \mathrm{~nm}\right)$ e Azul de Metileno $(319,8$ $\mathrm{g} \mathrm{mol}^{-1}, \lambda_{\max }=664 \mathrm{~nm}$ ) foram adquiridos da Vetec Química Fina Ltda., Brasil. Todos os outros reagentes utilizados foram de grau analítico. Prepararam-se soluções estoque dos corantes $\left(1,00 \mathrm{~g} \mathrm{~L}^{-1}\right)$ com água desionizada e as experiências subsequentes foram feitas por diluição destas. As estruturas moleculares dos corantes RB5 e MB estão apresentadas na Figura 2.

Figura 2 - Estrutura molecular do (a) RB5 e (b) MB.<smiles>COC(=O)CCS(=O)(=O)c1ccc(N=Nc2c(O)c(N)c(N=Nc3ccc(S(=O)(=O)CCOC(C)=O)cc3)c(O)c2[N+](=O)[O-])cc1</smiles>

(a)<smiles></smiles>

(b) 


\subsection{Ensaios de Adsorção}

Os experimentos de adsorção foram realizados em batelada a $200 \mathrm{rpm}$ utilizando um agitador termostático (SHAKER SL 222 - SOLAB), para verificação das curvas cinéticas. A dosagem de adsorvente e o $\mathrm{pH}$ adequado para cada corante foram definidos em ensaios preliminares. Os experimentos cinéticos foram realizados com $0,025 \mathrm{~g}$ de adsorvente, utilizando pH 2 para o RB5 e pH 8 para o MB. A concentração inicial dos corantes foi de 50 $\mathrm{mg} \mathrm{L}^{-1}$ e o volume de solução foi de $25 \mathrm{~mL}$. Os ensaios foram realizados a $25^{\circ} \mathrm{C}$ com tempo de contato variando de 0 a $240 \mathrm{~min}$. Após os experimentos, todas as amostras obtidas foram filtradas e a concentração remanescente dos corantes na fase líquida foi determinada por espectrofotômetro (UV mini 1240-SHIMADZU). A capacidade de adsorção no tempo $\left(q_{t}, m g\right.$ $\left.g^{-1}\right)$ foi calculada pela Equação 1 e a capacidade de adsorção no equilíbrio $\left(q_{e}, m g g^{-1}\right)$ foi calculada pela Equação 2 .

$$
\begin{aligned}
& q_{t}=\frac{\left(C_{0}-C_{t}\right)}{m} V \\
& q_{e}=\frac{\left(C_{0}-C_{e}\right)}{m} V
\end{aligned}
$$

Onde, $C_{0}, C_{e}$ e $C_{t}\left(\mathrm{mg} \mathrm{L}^{-1}\right)$ são, respectivamente, as concentrações dos corantes no início, no equilíbrio e no tempo, $m$ (g) é a massa de adsorvente e $V(\mathrm{~L})$ é o volume da solução.

\subsection{Modelo Cinético}

Para estudar o comportamento cinético de adsorção, as curvas experimentais foram ajustadas ao modelo denominado HSDM (Homogeneous Surface Diffusion Model). As equações matemáticas do modelo HSDM para geometria cilíndrica são:

$$
\begin{aligned}
& \rho_{p} \frac{\partial q}{\partial t}=\frac{1}{r} \frac{\partial}{\partial r}\left[r\left(\rho_{p} D_{s} \frac{\partial q}{\partial r}\right)\right] \\
& t=0,0 \leq r \leq R, q=0 \\
& \left.\frac{\partial q}{\partial r}\right|_{r=0}=0 \\
& q_{r=R}=\alpha C_{s} \\
& m \frac{d q}{d t}=V \frac{d C_{t}}{d t}
\end{aligned}
$$

Onde $\rho_{p}$ é a massa específica do adsorvente $\left(\mathrm{kg} \mathrm{m}^{-3}\right), D_{s}$ é o coeficiente de difusão $\left(\mathrm{m}^{2} \mathrm{~s}^{-1}\right)$ e $r$ é o raio da partícula cilíndrica $(\mathrm{m})$. 


\section{RESULTADOS}

\subsection{Caracterização do Adsorvente}

A Figura 3(a) apresenta o espectro FTIR das fibras de sisal e a Figura 3(b) apresenta as imagens de MEV.

Figura 3 - (a) FTIR e (b) MEV das fibras de sisal.

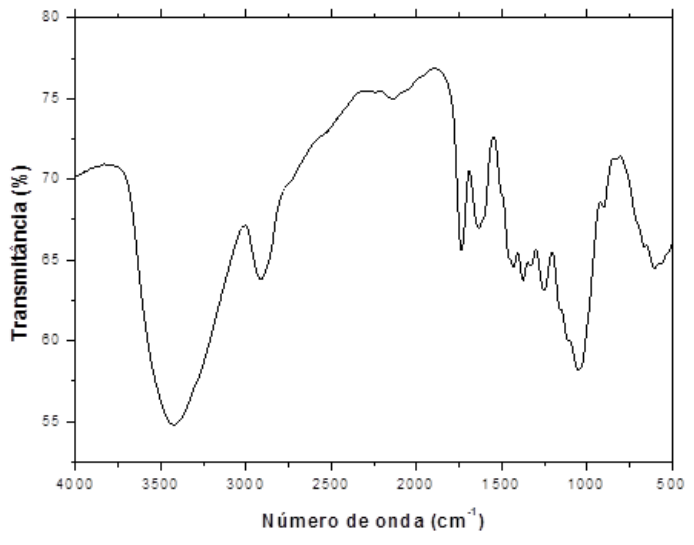

(a)

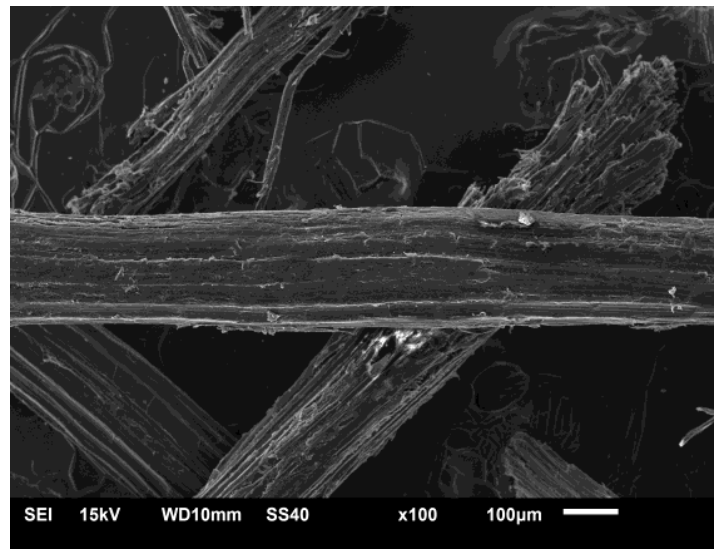

(b)

A Figura 3 (a) mostra que as principais bandas na fibra de sisal foram verificadas em $3300,2950,1750$ e na região de $1500-1000 \mathrm{~cm}^{-1}$. A banda intensa em $3300 \mathrm{~cm}^{-1}$ pode ser atribuída ao estiramento de ligações $\mathrm{OH}$ presentes na lignina, celulose ou hemicelulose. A banda em $2950 \mathrm{~cm}^{-1}$ é relativa a vibrações simétricas ou assimétricas de $-\mathrm{CH}_{2}$. A banda em $1750 \mathrm{~cm}^{-1}$ está relacionada com o $\mathrm{C}=\mathrm{O}$ da carbonila, que juntamente com o ombro ao redor de $1100 \mathrm{~cm}^{-1}$ sugere a presença de ácidos, ésteres ou lactonas ligadas a anéis aromáticos. A banda na região de $1000 \mathrm{~cm}^{-1}$ é relativa aos estiramentos de $\mathrm{C}-\mathrm{O}$ da lignina, celulose ou hemicelulose ou ainda C-O-C da celulose ou hemicelulose. Na região de $1500-1000 \mathrm{~cm}^{-1}$ podemos observar as vibrações de anéis guaiacílicos da lignina. Estes grupos funcionais podem ser potencias sítios para a adsorção de corantes, fazendo da fibra de sisal uma alternativa viável. A Figura 3 (b) mostra o aspecto fibroso do adsorvente. O material é formado de fibras maiores, as quais são compostas por fibrilas menores.

\subsection{Modelagem Cinética}

A cinética de adsorção fornece informações úteis sobre a eficiência de adsorção e viabilidade das operações realizadas. As curvas cinéticas do MB e RB5 estão apresentadas, respectivamente, nas Figuras 4 e 5. A partir da Figura 4 pode-se observar que, para o MB, a adsorção é muito rápida, sendo que a concentração final de corante torna-se praticamente constante em torno de 20 minutos, quando o equilíbrio é atingido. Já na adsorção do RB5, Figura 5, a adsorção é mais lenta, de tal forma que o equilíbrio só é atingido em torno de 150 minutos. A capacidade de adsorção no equilíbrio $\left(q_{e}\right)$, para o AM e para o RB5, respectivamente foi de aproximadamente $87 \mathrm{mg} \mathrm{g}^{-1}$ e de $29,8 \mathrm{mg} \mathrm{g}^{-1}$. Os percentuais de remoção foram maiores do que $85 \%$. 
Figura 4 - Cinética de adsorção do Azul de Metileno

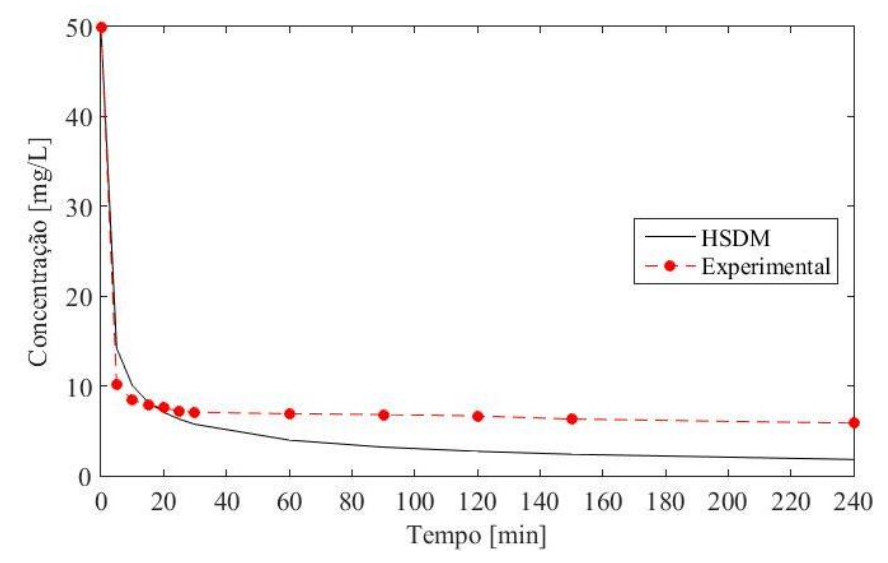

Figura 5 - Cinética de adsorção do Preto Reativo 5

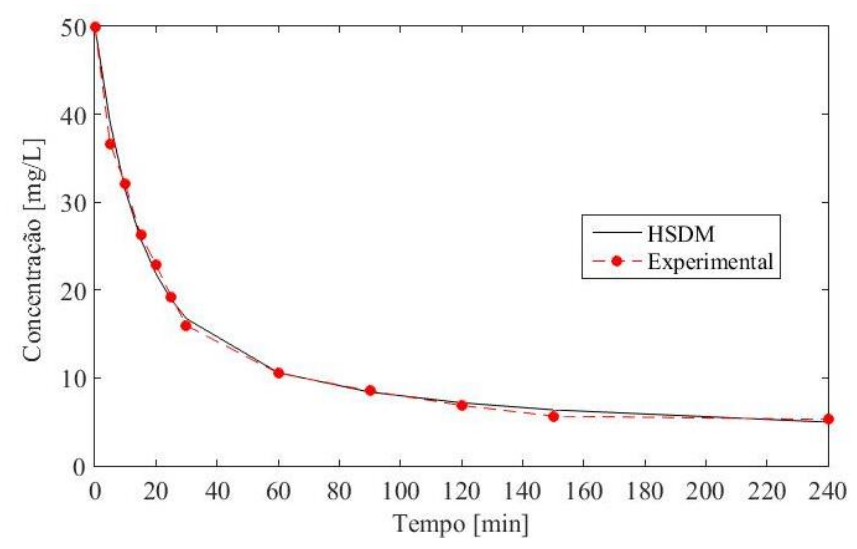

Para maior entendimento do comportamente cinético, os dados experimentais foram ajustados ao modelo HSDM. O modelo foi implementado e resolvido utilizando o software MatLab®. A solução numérica do modelo requeriu a aplicação do método numérico das linhas para a resolução da equação diferencial parcial (EDP) (Equação 5) que pode ser expressa como um sistema de equações diferenciais ordinárias (EDOs) simultâneas (Capeloneto e Buarque, 2016). Devido à rigidez das EDOs resultantes, a integração numérica do modelo foi realizada com um método numérico baseado em fórmulas de diferenciação numérica. Os parâmetros $D_{s}$ (Equação 5) e $\alpha$ (Equação 8) foram estimados através da otimização de mínimos quadrados não-lineares. A função objetivo a ser minimizada foi definida como a soma das diferenças entre as concentrações dos corantes experimentais $\left(C_{t}\right) \mathrm{e}$ as concentrações dos corantes obtidas pelo modelo $\left(\hat{C}_{t}\right)$, como segue na Equação 10:

$$
\min |f(p)|=\sum_{i=1}^{N Y}\left[C_{i}-\hat{C}_{i}\right]
$$

Onde $N Y$ é o número total de pontos experimentais e $p$ são os parâmetros a serem estimados. 
Os parâmetros estimados do modelo e os parâmetros de ajuste estão apresentados na Tabela 1.

Tabela 1. Parâmetros cinéticos na adsorção dos corantes pelas fibras de sisal.

\begin{tabular}{ccc}
\hline \multicolumn{3}{c}{ Modelo Cinético HSDM } \\
\hline Parâmetros & RB5 & MB \\
\hline$D s\left(\mathrm{~cm}^{2} \mathrm{~s}^{-1}\right)$ & $1,6 \times 10^{-11}$ & $5,9 \times 10^{-10}$ \\
$\alpha\left(\mathrm{L} \mathrm{mg}^{-1}\right)$ & 5,079 & 1,998 \\
$R^{2}$ & 0,9981 & 0,9824 \\
$R^{2}{ }_{d d j}$ & 0,9979 & 0,9806 \\
$A R E(\%)$ & 18,57 & 5,28 \\
$A I C$ & 89,7 & 39,9 \\
\hline
\end{tabular}

De acordo com a Tabela 1, a partir dos valores elevados do coeficiente de determinação $\left(R^{2}\right)$, muito próximos de 1 , e os baixos valores do erro médio relativo $(A R E)$, e critério de informação Akaike (AIC) observa-se que os dados experimentais foram bem ajustados pelo modelo cinético HSDM. A partir dos valores encontrados para o coeficiente difusivo, tem-se que para o $\mathrm{MB}$ o $D s$ estimado é maior do que o $D s$ estimado para o RB5, logo, o $\mathrm{MB}$ se difunde mais rapidamente dentro da partícula adsorvente. Este fato pode ser explicado devido à estrutura dos corantes. Como o Azul de Metileno possui estrutura mais simplificada, sua mobilidade ao longo da partícula adsorvente é facilitada, o que garante uma cinética de adsorção mais rápida. Estes resultados estão de acordo com as curvas cinéticas.

\section{CONSIDERAÇÕES FINAIS}

No presente trabalho, avaliou-se o potencial de fibras de sisal na remoção de corantes de solução aquosa por meio da adsorção, em termos de modelagem cinética. Os ensaios foram realizados com o corante aniônico Reativo Preto 5 (RB5) e catiônico Azul de Metileno (AM). A partir do ajuste dos dados experimentais ao modelo HSDM, verificou-se que a adsorção dos dois corantes é bem representada por este modelo. A cinética de adsorção do MB demonstrou-se mais rápida, sendo o equilíbrio atingido mais rapidamente do que no caso do RB5. Os valores do coeficiente de difusão $(D s)$ foram de $1,6 \times 10^{-11}$ e $5,9 \times 10^{-10} \mathrm{~cm}^{2} \mathrm{~s}^{-1}$ para os corantes preto reativo 5 (RB5) e azul de metileno(MB). As capacidades de adsorção no equilíbrio (qe), para o AM e para o RB5, respectivamente, foram de $87 \mathrm{mg} \mathrm{g}^{-1}$ e $29,8 \mathrm{mg} \mathrm{g}^{-1}$, com percentuais de remoção maiores que $85 \%$.

\section{REFERÊNCIAS BIBLIOGRÁFICAS}

CAPELONETO, J; BUARQUE, N.M.S. Simulation of saxitoxins adsorption in full-scale GAC filter using HSDM. Water Research, [s.1.], v. 88, p.558-565, jan. 2016.

VADIVELAN, V; KUMAR, K.V. Equilibrium, kinetics, mechanism, and process design for the sorption of methylene blue onto rice husk. Journal Of Colloid And Interface Science. 286 90-100,abr. 2005. 